\title{
A Novel Method for Recognition of Bioradiolocation Signal Breathing Patterns for Noncontact Screening of Sleep Apnea Syndrome
}

\author{
Maksim Alekhin (D), ${ }^{1}$ Lesya Anishchenko ${ }^{(D)},{ }^{1}$ Alexander Tataraidze ${ }^{(D)},{ }^{1,2}$ Sergey Ivashov $\left(\mathbb{D},{ }^{1}\right.$ \\ Vladimir Parashin, ${ }^{2}$ Lyudmila Korostovtseva ${ }^{(D},{ }^{3}$ Yurii Sviryaev, ${ }^{3}$ and Alexey Bogomolov ${ }^{4}$ \\ ${ }^{1}$ Remote Sensing Laboratory, Bauman Moscow State Technical University, Moscow 105005, Russia \\ ${ }^{2}$ Department of Biomedical Engineering, Bauman Moscow State Technical University, Moscow 105005, Russia \\ ${ }^{3}$ Sleep Laboratory, Almazov Federal Heart, Blood and Endocrinology Centre, Saint Petersburg 197341, Russia \\ ${ }^{4}$ State Research and Testing Institute of Military Medicine of the Ministry of Defense of Russia, Moscow 127083, Russia \\ Correspondence should be addressed to Maksim Alekhin; maksim.alekhin@gmail.com
}

Received 10 March 2013; Accepted 9 June 2013

Academic Editor: Francesco Soldovieri

Copyright (C) 2013 Maksim Alekhin et al. This is an open access article distributed under the Creative Commons Attribution License, which permits unrestricted use, distribution, and reproduction in any medium, provided the original work is properly cited.

\begin{abstract}
A novel method for recognition of breathing patterns of bioradiolocation signals breathing patterns (BSBP) in the task of noncontact screening of sleep apnea syndrome (SAS) is proposed and implemented on the base of wavelet transform (WT) and neural network (NNW) applications. Selection of the optimal parameters of WT includes determination of the proper level of wavelet decomposition and the best basis for feature extraction using modified entropy criterion. Selection of the optimal properties of NNW includes defining the best number of hidden neurons and learning algorithm for the chosen NNW topology. The effectiveness of the proposed approach is tested on clinically verified database of BRL signals corresponding to the three classes of breathing patterns: obstructive sleep apnea (OSA); central sleep apnea (CSA); normal calm sleeping (NCS) without sleep-disordered breathing (SDB) episodes.
\end{abstract}

\section{Introduction}

One of the critical areas of sleep medicine is implementation of novel technical approaches for remote vital signs monitoring [1], particularly in screening of sleep disordered breathing (SDB), which is character for sleep apnea syndrome (SAS) [2]. Early detection and proper classification of SDB episodes are an important aspect of SAS treatment strategy planning and taking of opportune preventive measures in clinical practice [3].

Bioradiolocation (BRL) [4] is a modern remote sensing technique allowing to perform noncontact vital signs monitoring of living objects [5] on the base of analysis of specific biometric modulation in reflected radiolocation signal. During tidal breathing process the modulation is mostly determined by reciprocating displacements of skin surface in abdominal and thoracic areas of chest wall due to periodic contractions of respiratory muscles [6]. The reliability and effectiveness of BRL technology application in noncontact respiratory monitoring [7] and remote screening of SAS [8] on the base of breathing pattern analysis were convincingly demonstrated.

Synthesis of intellectual radar data processing systems for target tracking, localization, and recognition is an up to date task of modern cybernetics. Methods and algorithms for automated recognition of patterns in nonstationary BRL signals are in demand in complex ergatic and biomedical systems [9].

Practically, improvement of performance of pattern recognition can be achieved by applying one of the following approaches or their combinations: firstly, by optimizing decision rules; secondly, by applying more efficient feature extraction methods [10]. Decision rules implemented on the base of neural networks (NNW) technology are considered to 


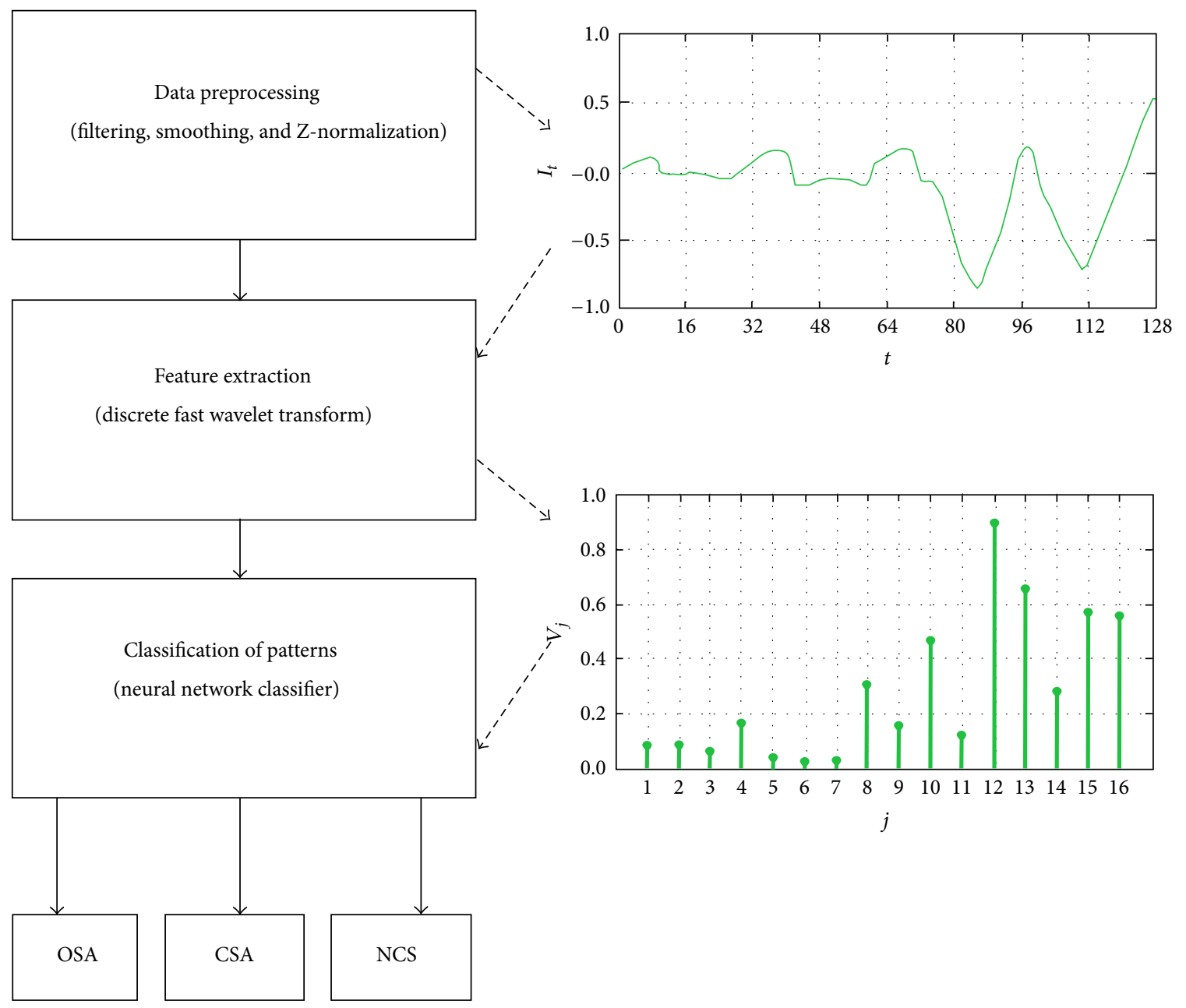

FIGURE 1: Scheme of the proposed method for recognition of BPBS on the base of WT and NNW.

be promising in practical applications of pattern recognition tasks [11]. In turn, wavelet transform (WT) is a perspective mathematical apparatus, which is especially demanded in forming of attribute space of nonstationary multicomponent signals with noise, for which high-frequency components of short duration and extended low-frequency components are typical [12].

The aim of this study is development of a novel method for recognition of breathing patterns of bioradiolocation signals (BPBS) in the task of noncontact screening of SAS with automated selection of optimal parameters of WT and NNW. The proposed approach is tested in recognition of clinically verified BPBS corresponding to the following classes: obstructive sleep apnea (OSA); central sleep apnea (CSA); normal calm sleeping (NCS) without SDB.

\section{Structure of the Proposed Pattern Recognition Method}

The proposed method for recognition of BPBS in noncontact screening of SAS includes the following main steps (Figure 1): (i) data preprocessing procedure (including high-pass and low-pass Butterworth filtering, resampling, smoothing with five-point moving average filter, and Z-normalization);

(ii) feature extraction (forming of BPBS attribute vectors consisting of mean-squared values of WT detailed coefficients of each BRL signal quadrature);

(iii) classification of patterns (applying the fixed number of resulting components of BPBS attribute vectors to the inputs of NNW classifier-Rumelhart multilayer perceptron (MLP) [13] — as an output comes the value corresponding to one of the target classes: OSA, CSA, and NCS).

For feature extraction an attribute space of absolute values of WT detailed coefficients for both BRL signal quadratures was constructed. Thereby each component of BPBS attribute vector on the chosen wavelet decomposition level can be calculated as follows:

$$
V_{j}=\sqrt{\left(d_{j}^{Q}\right)^{2}+\left(d_{j}^{I}\right)^{2}},
$$




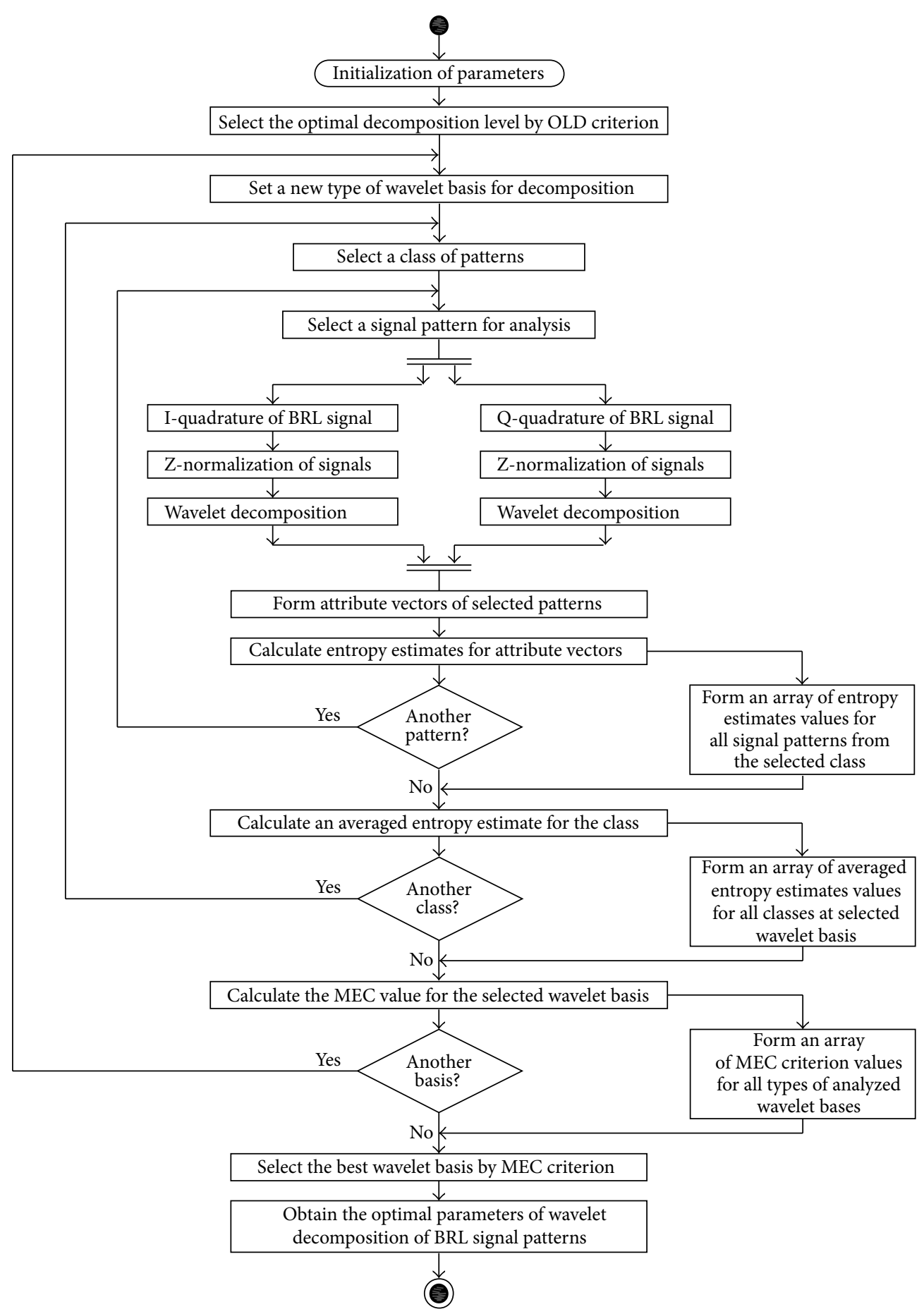

FIGURE 2: Scheme of the algorithm for automated selection of the optimal parameters of WT for improvement of BPBS feature extraction.

where $V_{j}$ is a resulting component of BPBS attribute vector; $j$ is current number of a resulting component of BPBS attribute vector; $d_{j}^{Q}$ is the detailed wavelet coefficient for $Q$-quadrature of BRL signal; $d_{j}^{I}$ is the detailed wavelet coefficient for $I$ quadrature of BRL signal.

For improvement of the performance of the proposed BSBP recognition method (Figure 1), procedures for both automated selection of the optimal WT parameters (Figure 2) and NNW properties (Figure 3) were developed.

\section{Optimization Criterions}

3.1. Criterion of the Optimal Level of Wavelet Decomposition. The estimate $f_{m}$ of the upper frequency limit for the range in 


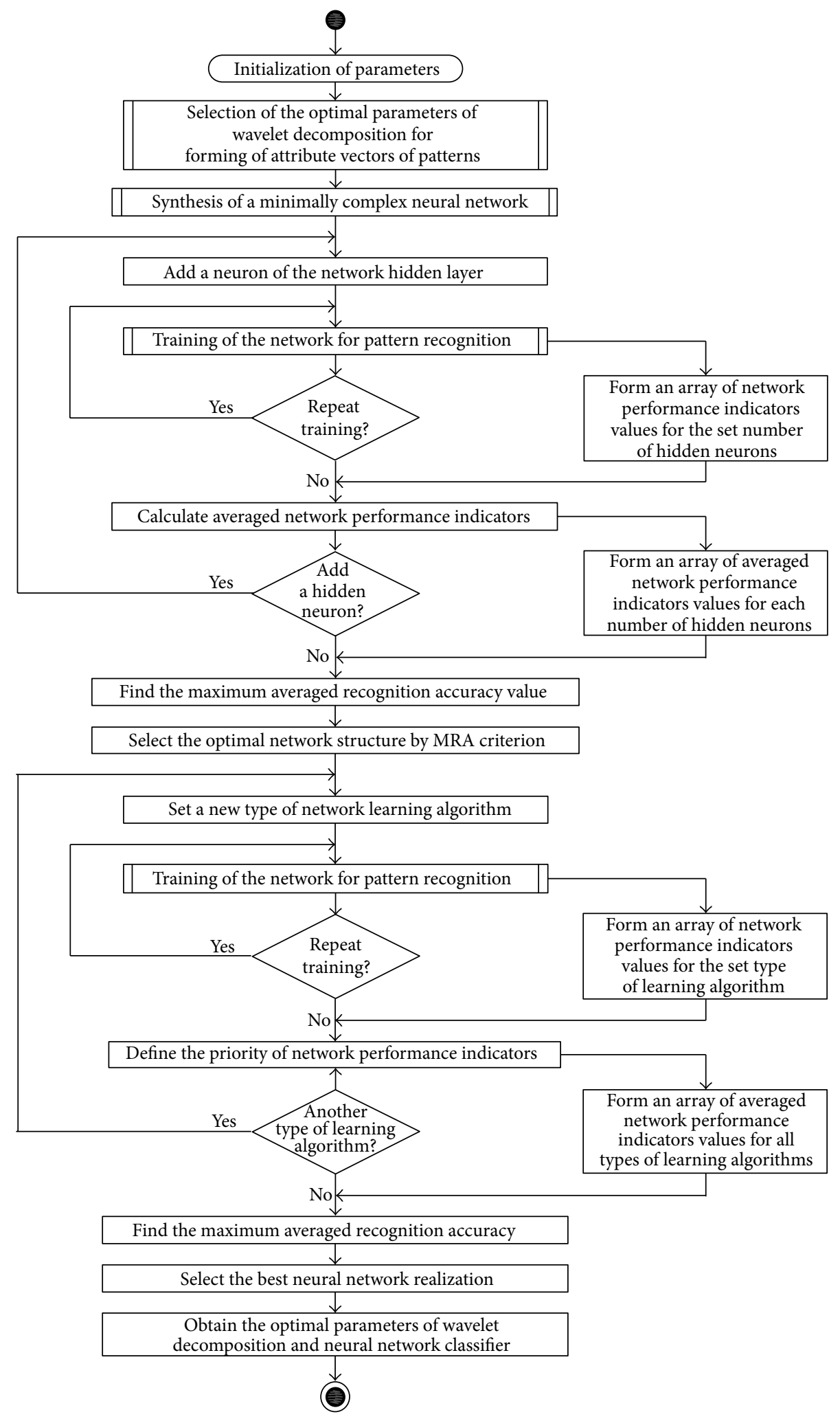

FIGURE 3: Scheme of the algorithm for automated selection of the optimal parameters of NNW for improvement of BPBS classification. 
which most of quasi-stationary signal energy is concentrated (the upper limit of normal breathing frequency range) is considered to be known for BRL signals [4]. The maximum possible frequency value for the registered signal is calculated in accordance with Nyquist theorem (is half the sampling rate $f_{s}$ ). Then the optimal decomposition level (ODL) of WT for an analyzed BRL signal can be calculated from the relation [14]:

$$
L_{o}=\left[\log _{2}\left(\frac{f_{s}}{2 f_{m}}\right)\right]+1=-\left[\log _{2}\left(2 f_{m} \Delta t\right)\right]+1,
$$

where $f_{m}$ is the upper limit of frequency band in which the most of signal energy is concentrated; $f_{s}$ is sampling rate; $\Delta t$ is sampling period.

Thus, further decomposition of analyzed BRL signals to the levels exceeding the threshold of ODL is not effective as calculated detailed coefficients of WT will not be informative in the aspect of effective feature extraction for BPBS attribute space constructing.

3.2. Criterion of the Optimal Basis of Wavelet Transform. For selection of the optimal basis of WT from the class of orthogonal wavelets with compact support, a modified entropy based criterion (MEC) is proposed which is calculated on the base of logarithm energy entropy estimation in the task of classification of BPBS:

$$
\begin{aligned}
E_{o} & =-\sqrt{\frac{1}{C N}\left(\sum_{k=1}^{C}\left(\sum_{i=1}^{N}\left(\sum_{j=1}^{K} \ln \left(\sqrt{\left(d_{j}^{\mathrm{Q}}\right)^{2}+\left(d_{j}^{I}\right)^{2}}\right)\right)\right)\right)} \\
& \longrightarrow \min ,
\end{aligned}
$$

where $E_{o}$ is estimate of MEC; $C$ is number of classes of patterns; $N$ is number of patterns in each class; $K$ is number of resulting components in attribute vectors; $\ln \left(d_{j}^{2}\right)$ is logarithm energy entropy estimate; $d_{j}^{\mathrm{Q}}$ is the detailed wavelet coefficient for Q-quadrature of BRL signal; $d_{j}^{I}$ is the detailed wavelet coefficient for $I$-quadrature of BRL signal.

Selection of the optimal basis of WT for effective BPBS attribute space forming should be performed using mean squared values of detailed wavelet coefficients of each BRL signal quadrature for MEC calculations.

3.3. Criterion of the Optimal Number of Hidden Neurons. For selection of the optimal number of hidden neurons of MLP for BPBS, recognition the mean recognition accuracy (MRA) criterion is proposed. Varying in each NNW operation test the number of hidden neurons, an estimate of MRA is calculated as follows:

$$
A_{o}=\frac{1}{M B}\left(\sum_{m=1}^{M}\left(\sum_{b=1}^{B} C_{b}\right)\right) \longrightarrow \max ,
$$

where $A_{o}$ is estimate of MRA; $M$ is number of operation tests; $B$ is number of analyzed wavelet basis; $C_{b}$ is recognition accuracy for analyzed wavelet basis.

Selection of the optimal number of hidden neurons of MLP with application of MRA criterion should be performed using wavelet bases with such ordinal indexes for which the minimal values of MEC are achieved on training data set.

\section{Optimization Algorithm}

4.1. Structure of the Optimization Algorithm. The proposed algorithm for automated selection of optimal parameters of WT and NNW for improving the performance of BPBS recognition consists of the two main steps. In the first step, for informative feature extraction applying WT, initially a general class of wavelets is defined, then a set of wavelet bases with ordinal indexes for wavelet families from the general class is formed, the optimal level of wavelet decomposition is determined, and finally the optimal wavelet basis is selected on the base of MEC. In the second step, for improving NNW operation performance, after preliminary estimation of number of hidden neurons such their optimal amount is found for which the best MRA value is achieved on training data set, and then the best NNW learning algorithm is selected.

4.2. Procedure for Selection of the Optimal Parameters of Wavelet Transform. The proposed procedure for selection of the optimal parameters of WT includes the following main steps (Figure 2):

(i) setting initial parameters characterized by the analyzed BRL signal properties (the maximum possible frequency of signal; sampling rate; and others);

(ii) defining general class of wavelets based on desirable properties for BRL signal processing (orthogonal wavelets with compact support-proper timefrequency localization and available fast algorithms for implementing WT);

(iii) forming a set of bases with ordinal indexes from wavelet families (Dobeshi, Coiflet, and Symlet familiesthe properties of orthogonality, regularity, symmetry, and approximating power of scaling functions depend on the ordinal index of the wavelet basis);

(iv) calculating the optimal level of wavelet decomposition (estimation of ODL threshold on the base of the maximum possible frequency of the analyzed BRL signal and sampling rate values);

(v) selecting the optimal wavelet basis (estimation of MEC on the base of log energy entropy value for the task of BPBS classification).

4.3. Procedure for Selection of the Optimal Parameters of Neural Network. The proposed procedure for selection of optimal parameters of NNW classifier on the base of MLP with nonlinear activation function includes the following main steps (Figure 3):

(i) setting initial parameters characterized by the conditions of the classification task (dimensions of input and output vectors, recognition accuracy, error values, and others); 


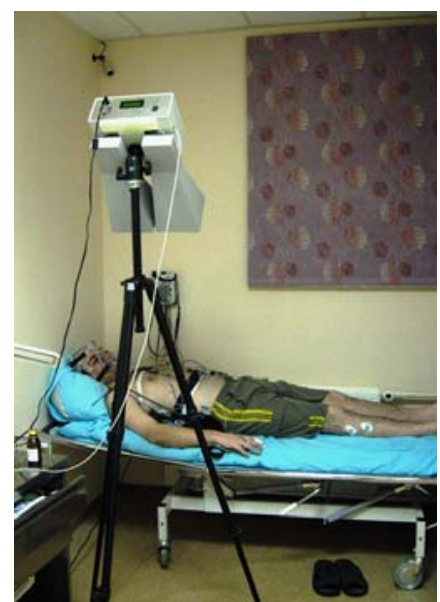

FIGURE 4: Scheme of the experiment on simultaneous registration of BRL and PSG data during sleep.

(ii) setting the number of hidden layers (one layer-the minimal complexity, availability of wide range of optimization, and learning algorithms);

(iii) estimating the upper boundary limit of hidden neurons number (in accordance with KolmogorovHecht-Nielsen theorem [15] for MLP);

(iv) choosing structural optimization method (simple iterative algorithm of adding extra neurons);

(v) defining the optimal number of hidden neurons (calculation of MRA during several operation tests of NNW on a training data set);

(vi) selecting the best NNW learning algorithm (on the base of such performance indicators as recognition accuracy, time needed, computational complexity, and others).

\section{Experimental Study}

For the test and optimization of the proposed methods and algorithms for automated recognition of BPBS, the clinically verified database of BRL signals for subjects with SAS collected during simultaneous registration of full-night polysomnography (PSG) acquired in the Sleep Laboratory of Almazov Federal Heart, Blood and Endocrinology Centre (Figure 4) was used [8]. The sample included 7 subjects ( 4 males and 3 females, aged 43-62 years, with body mass index of 21.6-57.7), depending on severity of SAS: 4 severe; 1 moderate; 1 mild; 1 normal. The full-night PSG records were collected with Embla N7000 system. Simultaneously BioRascan multifrequency BRL system with a continuouswave signal and step frequency modulation (developed at Remote Sensing Laboratory of Bauman Moscow State Technical University) was used applying the operating frequency range 3.6-4.0 GHz. The internal clock of BRL and PSG systems were synchronized for further verification [8].

For forming BPBS attribute vectors, both BRL signal quadratures were used according to (1) with the same length
TABLE 1: Selection of the optimal wavelet basis for BPBS feature extraction.

\begin{tabular}{lcccc}
\hline \multirow{2}{*}{ Wavelet basis } & \multicolumn{4}{c}{ Entropy estimates } \\
& $E_{1}$ & $E_{2}$ & $E_{3}$ & $E_{o}$ \\
\hline DBSH13 & $-31,840$ & $-70,530$ & $-35,636$ & $-\mathbf{4 9 , 1 8 8}$ \\
SMLT13 & $-\mathbf{3 2 , 7 9 0}$ & $-\mathbf{8 0 , 1 0 4}$ & $-\mathbf{4 4 , 9 6 4}$ & $-\mathbf{5 6 , 3 1 3}$ \\
CFLT5 & $-39,672$ & $-71,158$ & $-33,537$ & $-\mathbf{5 0 , 8 6 6}$ \\
\hline
\end{tabular}

Where $E_{1}, E_{2}$, and $E_{3}$ are averaged entropy estimates for the three classes of BPBS; $E_{o}$ is MEC estimates.

of 128 counts corresponding to 12.8 seconds satisfying the recommendations for screening of SAS [2]. In correspondence with (2) for BRL signals at sampling rate of $f_{s}=10.0 \mathrm{~Hz}$ with maximum breathing frequency [16] not exceeding the value of $f_{m}=1.0 \mathrm{~Hz}$, the OLD value cames to $L_{o}=3$ providing 16 components in the structure of BPBS attribute vectors.

The experimental data set included three classes of BPBS (Figure 5): obstructive sleep apnea (OSA); central sleep apnea (CSA); normal calm sleeping without SDB (NCS).

The experimental data set included 240 realizations of BPBS related to the three classes (OSA, CSA, and NCS) in the following proportion:

(i) 90 patterns (30 in each class) — training set;

(ii) 30 patterns (10 in each class) - validation set;

(iii) 120 patterns (40 in each class) - test set.

\section{Results and Discussion}

6.1. Selection of the Optimal Wavelet Basis. The optimal wavelet basis for constructing BPBS attribute space was selected from orthogonal wavelets with compact support, including the following ones available in MATLAB [12]:

(i) Dobeshi-16 bases (ordinal indexes from 1 to 16 );

(ii) Symlet-14 bases (ordinal indexes from 4 to 17);

(iii) Coiflet -5 bases (ordinal indexes from 1 to 5 ).

For each wavelet basis, BPBS attribute vectors were formed on the base of mean-squared values of WT detailed coefficients of each BRL signal quadrature according to (1). The results of the calculations of MEC estimates for the best bases from each family are given in Table 1.

Thus wavelet basis Symlet 13 should be considered the best according to MEC, but for the analysis of effectiveness and consistency of the proposed entropy criterion itself the best bases for Dobeshi and Coiflet wavelet families were also subsequently considered.

6.2. Selection of the Optimal Number of Hidden Neurons. After forming of attribute vectors of BPBS applying selected wavelet bases (Dobeshi 13, Symlet 13, and Coiflet 5), the training of NNW classifier was 10 times independently performed for each case varying the number of hidden neurons from 1 to 15. MRA values for the three wavelet bases at varied number of hidden neurons are given in Table 2 . 


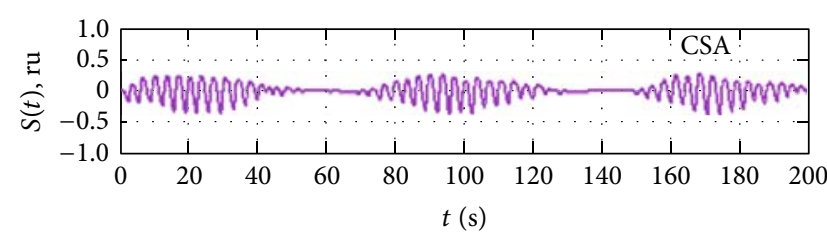

(a)

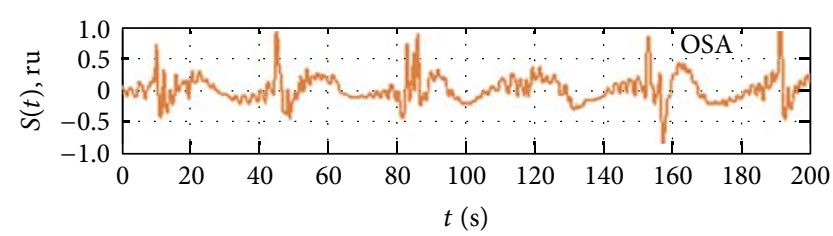

(b)

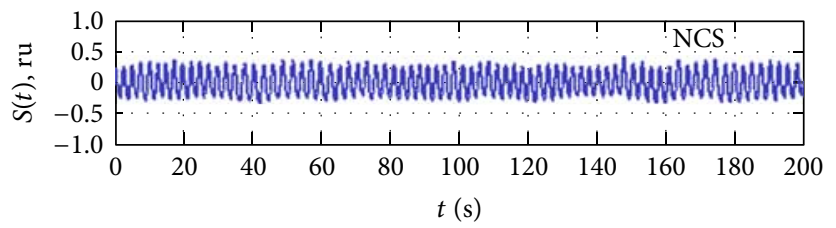

(c)

FIGURE 5: Typical BRL signals for the three classes of analyzed patterns (OSA, CSA, and NCS).

TABLE 2: Selection of the optimal number of hidden neurons for NNW.

\begin{tabular}{lccccc}
\hline \multirow{2}{*}{ Number of neurons } & \multicolumn{5}{c}{ Recognition accuracy values } \\
& $A_{\mathrm{DBSH} 13}$ & $A_{\text {SMLT13 }}$ & $A_{\text {CFLT }}$ & $A_{\text {Max }}$ & $A_{o}$ \\
\hline 1 & 0,360 & 0,455 & 0,479 & $\mathbf{0 , 4 7 9}$ & $\mathbf{0 , 4 3 1}$ \\
2 & 0,561 & 0,620 & 0,562 & $\mathbf{0 , 6 2 0}$ & $\mathbf{0 , 5 8 1}$ \\
3 & 0,732 & 0,675 & 0,746 & $\mathbf{0 , 7 4 6}$ & $\mathbf{0 , 7 1 8}$ \\
4 & 0,730 & 0,793 & 0,734 & $\mathbf{0 , 7 9 3}$ & $\mathbf{0 , 7 5 2}$ \\
5 & 0,789 & 0,823 & 0,756 & $\mathbf{0 , 8 2 3}$ & $\mathbf{0 , 7 8 9}$ \\
6 & 0,768 & 0,818 & 0,808 & $\mathbf{0 , 8 1 8}$ & $\mathbf{0 , 7 9 8}$ \\
7 & 0,799 & 0,842 & 0,814 & $\mathbf{0 , 8 4 2}$ & $\mathbf{0 , 8 1 8}$ \\
8 & 0,821 & 0,825 & 0,815 & $\mathbf{0 , 8 2 5}$ & $\mathbf{0 , 8 2 0}$ \\
$\mathbf{9}$ & $\mathbf{0 , 8 3 6}$ & $\mathbf{0 , 8 5 0}$ & $\mathbf{0 , 8 3 6}$ & $\mathbf{0 , 8 5 0}$ & $\mathbf{0 , 8 4 1}$ \\
10 & 0,813 & 0,848 & 0,833 & $\mathbf{0 , 8 4 8}$ & $\mathbf{0 , 8 3 1}$ \\
11 & 0,820 & 0,839 & 0,812 & $\mathbf{0 , 8 3 9}$ & $\mathbf{0 , 8 2 4}$ \\
12 & 0,821 & 0,831 & 0,825 & $\mathbf{0 , 8 3 1}$ & $\mathbf{0 , 8 2 6}$ \\
13 & 0,836 & 0,823 & 0,827 & $\mathbf{0 , 8 3 6}$ & $\mathbf{0 , 8 2 9}$ \\
14 & 0,788 & 0,820 & 0,818 & $\mathbf{0 , 8 2 0}$ & $\mathbf{0 , 8 0 9}$ \\
15 & 0,824 & 0,782 & 0,794 & $\mathbf{0 , 8 2 4}$ & $\mathbf{0 , 8 0 0}$ \\
\hline
\end{tabular}

Where $A_{\mathrm{DBSH} 13}, A_{\mathrm{SMLT1}}$, and $A_{\mathrm{CFLT} 5}$ are mean BPBS recognition accuracy values for corresponding wavelet bases (Dobeshi 13, Symlet 13, and Coiflet 5) at the set number of hidden neurons of NNW; $A_{\mathrm{Max}}$ is the maximum BPBS recognition accuracy value; $A_{o}$ is MRA estimate.

Thus according to MRA criterion, the optimal number of hidden neurons of NNW should be considered equal to 9 which also corresponds to the upper boundary limit estimated from Kolmogorov-Hecht-Nielsen theorem for MLP [15]. Herewith the best absolute value of recognition accuracy was also achieved for wavelet basis Symlet 13. The estimate (2) of MEC should also be considered effective and consistent as varying the number of hidden neurons from 1 to 15 at mean recognition accuracy not less than $75 \%$ for each analyzed wavelet, the basis of WT with the optimal MEC value allowed to achieve absolutely the best value of recognition accuracy value.
6.3. Selection of the Best Learning Algorithm. Previously for defining the optimal number of hidden neurons scaled conjugate gradient algorithm was used for training as it is considered to be the most multipurpose for feed forward NNW topologies [13]. Subsequently to select the best learning algorithm, the following gradient based variants implemented in MATLAB were considered [11]:

(i) first-order gradient (FOG) algorithms-resilient backpropagation (RPA);

(ii) conjugate gradient (CG) algorithms-FletcherReeves (FRA); Polak-Ribiére (PRA); Beale-Powell (BPA); Moller (MA);

(iii) quasi-Newton (QN) algorithms-Levenberg-Marquardt (LMA) Broyden-Fletcher-Goldfarb-Shanno (BFGSA); Battiti (BA).

Selection of the best learning algorithm was performed for MLP with one hidden layer with 9 hidden neurons with application of wavelet basis Symlet 13 on the 3rd level of wavelet decomposition in forming of BPBS attribute vectors.

Each time after 10 independent NNW operation tests on the test data set the following averaged performance indicators for learning algorithms were calculated: mean recognition accuracy, number of epochs needed for training, and time spent. Cross-validation criterion [11] was used to determine the moment to stop training. The results in order of priority of the algorithms are given in Table 3.

Thus LMA learning algorithm should be considered the best in training of NNW for BPBS recognition in the task of noncontact SAS screening, providing the mean accuracy not less than $84 \%$ with type II error not exceeding $8 \%$ for SDB episodes. The best absolute recognition accuracy of $86.7 \%$ was also achieved for NNW realization with LMA learning algorithm (Table 4).

\section{Conclusion}

A novel method for of BPBS recognition in the task of noncontact SAS screening with automated selection of optimal parameters of WT and NNW was developed. The BPBS 
TABLE 3: Selection of the best NNW learning algorithm.

\begin{tabular}{lcccc}
\hline \multicolumn{2}{c}{ Algorithm } & \multicolumn{3}{c}{ Performance indicators } \\
Rank & Title & Accuracy & Epochs & Time (ms) \\
\hline I & LMA & $\mathbf{0 , 8 4 2}$ & $\mathbf{1 2}$ & $\mathbf{4 9 3}$ \\
II & MA & $\mathbf{0 , 8 2 6}$ & 41 & 801 \\
III & BA & $\mathbf{0 , 8 1 1}$ & 39 & 936 \\
IV & RP & $\mathbf{0 , 8 0 2}$ & 29 & 592 \\
V & BPA & $\mathbf{0 , 7 9 8}$ & 25 & 757 \\
VI & BFGSA & $\mathbf{0 , 7 9 4}$ & 23 & 811 \\
VII & PRA & $\mathbf{0 , 7 9 3}$ & 21 & 683 \\
VIII & FRA & $\mathbf{0 , 7 5 4}$ & 26 & 648 \\
\hline
\end{tabular}

TABLE 4: Efficiency of BPBS recognition.

\begin{tabular}{lcccc}
\hline True & OSA & CSA & NCS & $\beta_{\text {err }}$ \\
\hline OSA & $\mathbf{3 6}$ & 0 & 3 & $6,9 \%$ \\
CSA & 1 & $\mathbf{3 5}$ & 2 & $7,2 \%$ \\
NCS & 6 & 4 & $\mathbf{3 3}$ & $20,8 \%$ \\
$\alpha_{\text {err }}$ & $15,2 \%$ & $9,5 \%$ & $10,4 \%$ & $\mathbf{8 6 , 7 \%}$ \\
\hline
\end{tabular}

attribute space was formed on the base of mean-squared values of wavelet detailed coefficients of each BRL signal quadrature. MLP with one hidden layer and nonlinear activation function was used as a classifier. The proposed method was tested on clinically verified database of BRL signals related to the three classes of BPBS: OSA; CSA; NCS.

In accordance with proposed MEC and ODL criterions, basis Symlet 13 from the general class of wavelets with compact support on the $3 \mathrm{rd}$ level of wavelet decomposition was considered to be the best one for feature extraction. The estimate of MEC itself should be considered effective and consistent. Calculation of MRA criterion revealed that the optimal number of NNW hidden neurons is equal to 9 which also corresponded to the upper boundary limit estimated from Kolmogorov-Hecht-Nielsen theorem. LMA should be considered the best training algorithm for the proposed classifier providing the mean recognition accuracy not less than $84 \%$ with type II error not exceeding $8 \%$.

\section{Acknowledgments}

The research was performed in the framework of the "Active and Passive Microwaves for Security and Subsurface imaging (AMISS)" EU 7th Framework Marie Curie Actions IRSES Project (PIRSES-GA-2010-269157) and supported by the grants of the Ministry of Education and Science of Russian Federation and Russian Foundation for Basic Research.

\section{References}

[1] F. Soldovieri, I. Catapano, L. Crocco, L. Anishchenko, and S. Ivashov, "A feasibility study for life signs monitoring via a continuous-wave radar," International Journal of Antennas and Propagation, vol. 2012, Article ID 420178, 5 pages, 2012.

[2] American Sleep Disorders Association Standards of Practice Committee, "Practice parameters for the indications for polysomnography and related procedures," Sleep, vol. 20, no. 6, pp. 406-422, 1997.

[3] L. Korostovtseva, Y. Sviryaev, N. Zvartau, A. Konradi, and A. Kalinkin, "Prognosis and cardiovascular morbidity and mortality in prospective study of hypertensive patients with obstructive sleep apnea syndrome in St. Petersburg Russia," Medical Science Monitor, vol. 17, no. 3, pp. 146-153, 2011.

[4] A. S. Bugaev, V. V. Chapursky, S. I. Ivashov, V. V. Razevig, A. P. Sheyko, and I. A. Vasilyev, "Through wall sensing of human breathing and heart beating by monochromatic radar," in Proceedings of the 10th International Conference Ground Penetrating Radar (GPR '04), pp. 291-294, Delft, The Netherlands, June 2004.

[5] S. Ivashov, V. Razevig, A. Sheyko, and I. Vasilyev, "Detection of human breathing and heartbeat by remote radar," in Proceedings of the Progress in Electromagnetics Research Symposium (PIERS '04), pp. 663-666, Pisa, Italy, March 2004.

[6] L. Liu, Z. Liu, and B. E. Barrowes, "Through-wall bioradiolocation with UWB impulse radar: observation, simulation and signal extraction," IEEE Journal of Selected Topics in Applied Earth Observations and Remote Sensing, vol. 4, no. 4, pp. 791-798, 2011.

[7] M. Alekhin, L. Anishchenko, A. Tataraidze, S. Ivashov, V. Parashin, and A. Dyachenko, "Comparison of bio-radiolocation and respiratory plethysmography signals in time and frequency domains on the base of cross correlation and spectral analysis," International Journal of Antennas and Propagation, vol. 2013, Article ID 410692, 6 pages, 2013.

[8] M. Alekhin, L. Anishchenko, A. Zhuravlev et al., "Estimation of information value of diagnostic data obtained by bioradiolocation pneumography in non-contact screening of sleep apnea syndrome," Biomedical Engineering, vol. 47, no. 2, pp. 96-99, 2013.

[9] S. Stergiopoulos, Advanced Signal Processing Handbook: Theory and Implementation for Radar, Sonar, and Medical Imaging Real Time Systems, CRC Press, New York, NY, USA, 2000.

[10] C. Bishop, Pattern Recognition and Machine Training, Springer, New York, NY, USA, 2006.

[11] I. Nabney, Netlab: Algorithms for Pattern Recognition, Springer, New York, NY, USA, 2001.

[12] M. Weeks, Digital Signal Processing Using MATLAB and Wavelets, Jones \& Bartlett Training, Boston, Mass, USA, 2nd edition, 2011.

[13] S. Haykin, Neural Networks: A Comprehensive Foundation, Prentice Hall, New York, NY, USA, 2nd edition, 1998.

[14] R. L. Kirlin and R. M. Dizaji, "Optimum sampling frequency in wavelet based signal compression," in Proceedings of the Canadian Conference on Electrical and Computer Egineering (CCECE '00), pp. 426-429, May 2000.

[15] R. Hecht-Nielsen, "Kolmogorov's Mapping Neural Network Existence Theorem," in Proceedings of the 3rd International Conference on Neural Networks, pp. 11-14, 1987.

[16] D. A. Korchagina, M. D. Alekhin, and L. N. Anishchenko, "Bioradiolocation method at chest wall motion analysis during tidal breathing," in Proceedings of the 7th European Radar Conference (EuRAD '10), pp. 475-478, Paris, France, October 2010. 

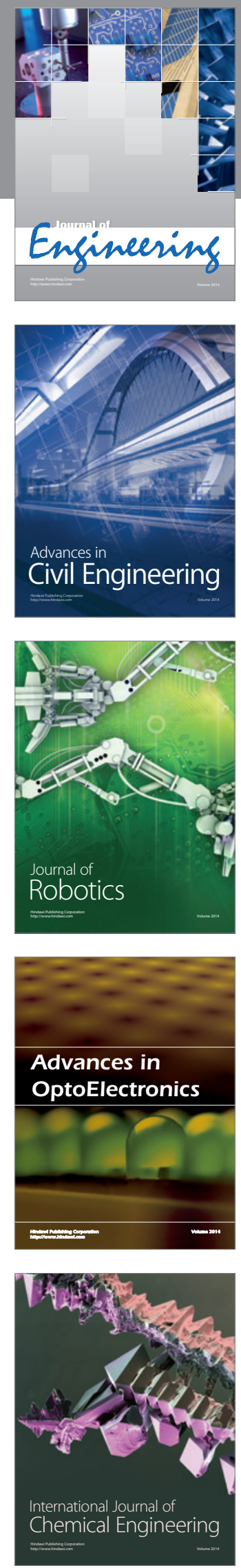

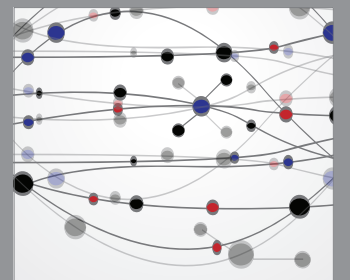

The Scientific World Journal
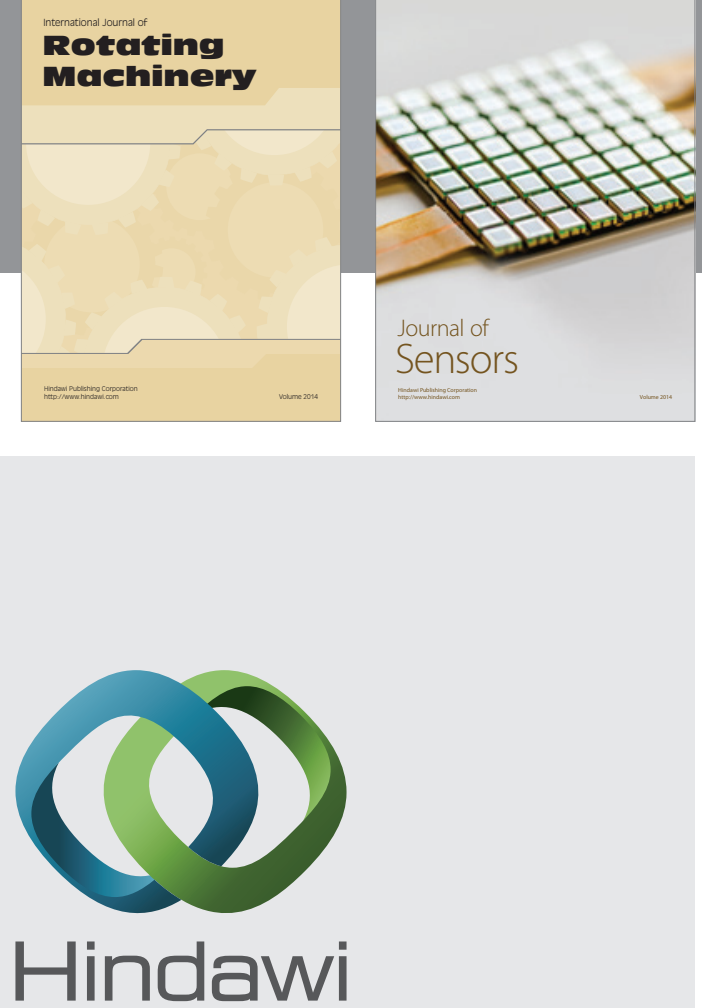

Submit your manuscripts at http://www.hindawi.com
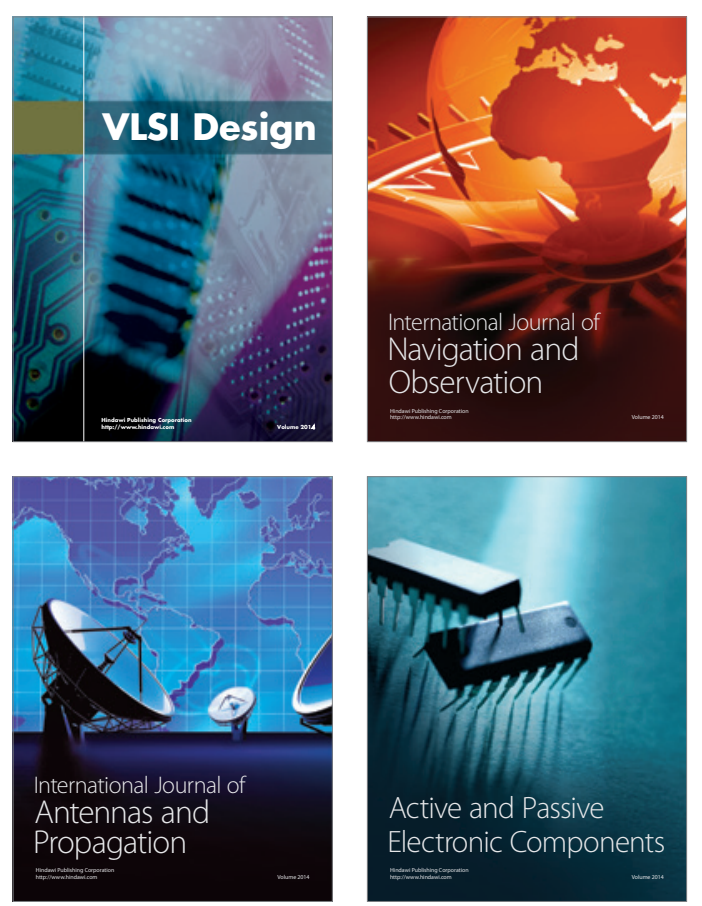
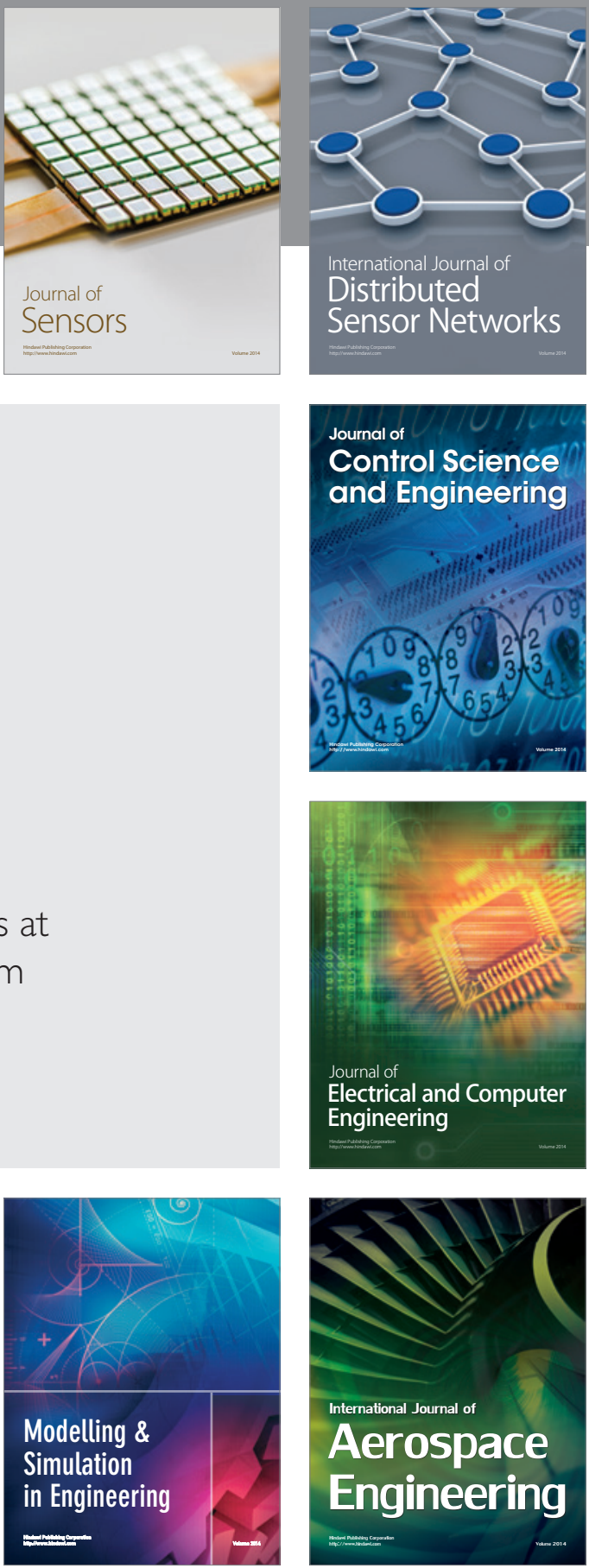

Journal of

Control Science

and Engineering
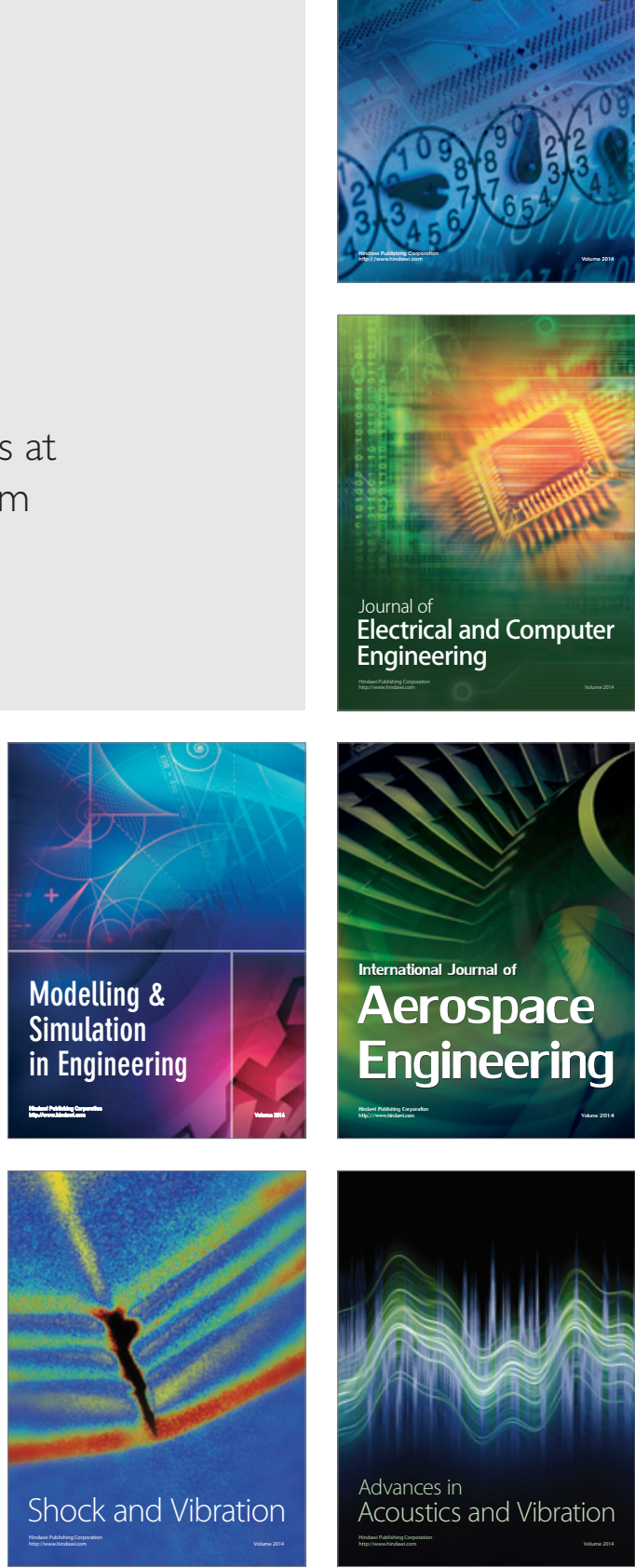